\title{
A Novel 0.3-0.31 THz GaAs-Based Transceiver with On-Chip Slotted Metamaterial Antenna Based on SIW Technology
}

\author{
Mohammad Alibakhshikenari ${ }^{1 *}$, Bal S. Virdee ${ }^{2}$, Chan H. See ${ }^{3,4}$, Raed Abd-Alhameed ${ }^{5}$, Francisco Falcone ${ }^{6}$, \\ and Ernesto Limiti ${ }^{1}$ \\ ${ }^{1}$ Electronic Engineering Department, University of Rome “Tor Vergata”, Via del Politecnico 1, 00133, Rome, ITALY \\ ${ }^{2}$ London Metropolitan University, Center for Communications Technology, London N7 8DB, UK \\ ${ }^{3}$ School of Engineering and the Built Environment, Edinburgh Napier University, 10 Colinton Road, Edinburgh, EH10 \\ 5DT, UK \\ ${ }^{4}$ School of Engineering, University of Bolton, Deane Road, Bolton, BL3 5AB, UK \\ ${ }^{5}$ School of Electrical Engineering \& Computer Science, University of Bradford, Bradford, BD7 1DP, UK \\ ${ }^{6}$ Electric and Electronic Engineering Department, Universidad Pública de Navarra, SPAIN \\ *alibakhshikenari@ing.uniroma2.it
}

\begin{abstract}
This paper presents a novel on-chip antenna with fully integrated $0.3-0.31$ THz transceiver is implemented on $0.5 \mu \mathrm{m}$ GaAs substrate, and comprises a voltage-controlled oscillator (VCO), a buffer amplifier, a modulator stage, a power-amplifier, a frequency-tripler, and an on-chip antenna. The proposed on-chip antenna design is based on metamaterial (MTM) slots and substrate integrated waveguide (SIW) technologies. The SIW antenna operates as a high-pass filter and an on-chip radiator to suppress the unwanted harmonics and radiate the desired signal, respectively. Dimensions of the on-chip antenna are $2 \times 1 \times 0.0006 \mathrm{~mm}^{3}$. The proposed on-chip antenna has an average radiation gain and efficiency of $>1.0 \mathrm{dBi}$ and $\sim 55 \%$, respectively. The transceiver provides an average output power of $-15 \mathrm{dBm}$ over $0.3-0.31 \mathrm{THz}$, which is suitable for near-field active imaging applications at terahertz region.
\end{abstract}

Keywords: On-chip antenna, metamaterial, terahertz (THz), substrate integrated waveguide (SIW), transceiver, Gallium Arsenide (GaAs) substrate, longitudinal and transverse slot arrays.

\section{INTRODUCTION}

High frequencies technologies in the millimetre-wave and terahertz bands have been successfully employed in various systems such as safety critical, biomedical, nondestructive detection, and non-ionizing cancer imaging [1, 2]. Such high frequency systems are fabricated using III-V semiconductor technology [3]. Although such systems can also be made from quasi-optical components [4] these are undesirable as they are physically large and uneconomic. Recent advances in complementary metal-oxidesemiconductor (CMOS) FETs and high-speed silicongermanium (SiGe) heterojunction bipolar transistors (HBTs) with significantly increased $f_{T}$ and $f_{\max }$ have resulted in the realization of single-chip integrated transmitters and receivers operating at millimetre-wave [5] and terahertz $(\mathrm{THz})$ bands [6-8].

This paper presents an investigation on a fully integrated on-chip antenna designed on GaAs based transceiver. The metamaterial slotted on-chip antenna is implemented on a SIW $[9,10]$ for integration within 300$310 \mathrm{GHz}$ transceiver in order to transmit the power efficiently as well as mitigate the unwanted harmonics. In the architecture of the transceiver, a frequency tripler and SIW slotted metamaterial on-chip antenna are conjugately impedance matched to improve the transceivers performance by reducing mismatching effects.

\section{TRANSCEIVER CIRCUIT DESIGN}

\section{A. Block Diagram}

The simplified architecture of the proposed $\mathrm{THz}$ transceiver shown in Fig. 1 is constituted from six components, i.e. a voltage-controlled oscillator (VCO), a buffer amplifier, a modulator stage, a power-amplifier, a frequency-tripler, and an on-chip antenna. The frequencytripler is necessary to generate the required terahertz frequency. This is achieved by using a $0.5 \mu \mathrm{m} \mathrm{GaAs}$ with $f_{T}$ and $f_{\max }$ of $150 \mathrm{GHz}$ and $220 \mathrm{GHz}$, respectively. The switchable modulator, which is fabricated on $0.5 \mu \mathrm{m}$ metal oxide semiconductor (MOS) FET, is incorporated in the transceiver architecture to enhance its functionality. Hence, the transceiver can be used for ultra-high data-rate $(\sim 10$ Gbps $)$ transmission or for highly accurate measurement applications including positioning and ranging, and Fourier-transform infrared spectroscopy (FTIR) where the modulation function needs to be disabled.

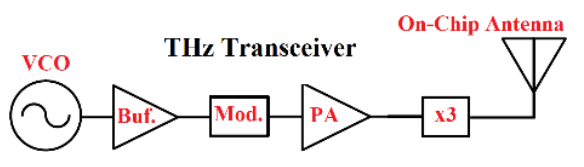

Fig.1. Schematic block diagram of the integrated terahertz transceiver attached to an on-chip SIW antenna.

\section{B. Voltage-Controlled Oscillator (VCO)}

The diagram in Fig. 2 is of the push-push cross-coupled VCO. The VCO's resonant frequency is controlled by inductance $(L)$ and capacitance $\left(C_{l}\right)$ of the varactor diode. The capacitance of the varactor diode is realized by joining HBT's emitter and base terminals and by using the collector-base junction in reverse bias mode. The total capacitance of the VCO is made up from the collector-base junction capacitance plus collector substrate parasitic capacitance. At the connection node of the HBTs $\left(Q_{l}\right.$ and $\left.Q_{2}\right)$ a $\lambda / 4$ microstrip-line $\left(T L_{1}\right)$ at $150 \mathrm{GHz}$ is attached in order to short-circuit only the second order harmonic. This has negligible effect on other signals. 


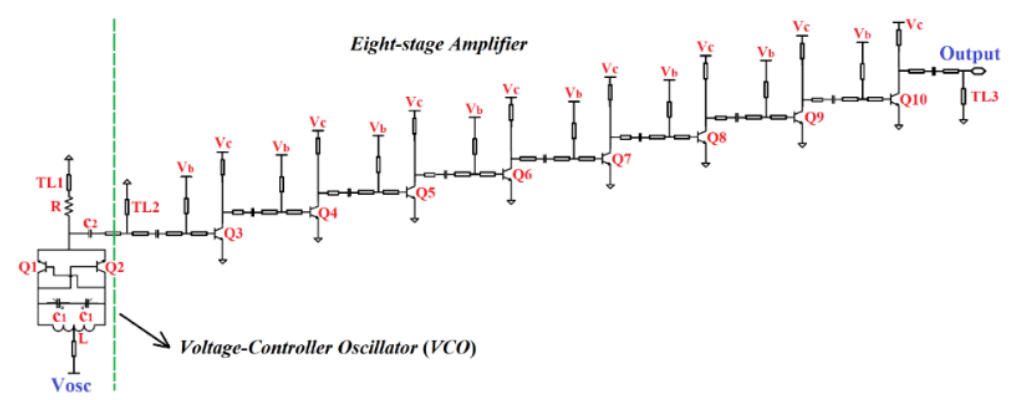

Fig.2. Circuit diagram of the voltage-controller oscillator (VCO) and the eight-stage amplifier.

\section{Buffer and Power Amplifier}

The circuit diagram of the eight-stage common-emitter amplifier configuration used for buffering and power amplification is shown in Fig. 2. To minimise parasitic effects and the footprint of the chip we have combined the bias network with the impedance matching network. The amplifier stages in the eight-stage amplifier were designed to be unconditionally stable over the frequency range of interest.

\section{Frequency Tripler $(\times 3)$}

Fig. 3 shows the circuit diagram of the frequencytripler. The frequency-tripler design uses nonlinear characteristics exhibited in HBT cascode $\left(Q_{1}\right.$ and $\left.Q_{2}\right)$. In conventional designs a bandpass filter is used to eliminate undesirable frequency components, i.e. fundamental and harmonic, at the output. At such high frequencies a bandpass filter is challenging to realise for low passband loss. Hence, we have adapted an open-stub $T L_{1}$ at $2 f_{0}$ to mitigate even harmonic components. The $L C$ network $\left(T L_{2}\right.$ and $C_{l}$ ), which is also used here in DC biasing, is used to eliminate the fundamental signal $\left(f_{0}\right)$ but allow $3 f_{0}$ signal.

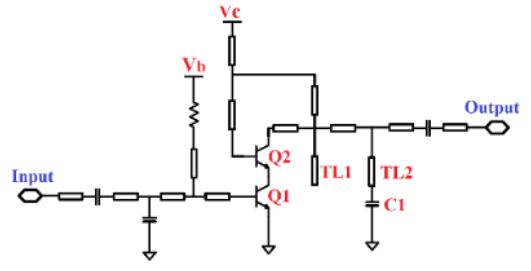

Fig.3. Circuit schematic of the frequency tripler.

The frequency-tripler was internally impedance matched to the on-chip SIW antenna. This was achieved by using conjugate matching in order to minimise the footprint of the circuit and to enhance the gain. Fig. 4 shows the simulated time-domain waveforms of the frequencytripler. It is evident that the $3^{\text {rd }}$ harmonic $\left(3 f_{0}\right)$ produced is significantly stronger. Fig. 4 also shows the fundamental signal $\left(f_{0}\right)$. It should be noted that the fundamental frequency is filtered out by the on-chip SIW antenna, which is based on metamaterial slots having high-pass characteristic.

\section{E. On-Chip Antenna Based on Substrate Integrated Waveguide and Metamaterial Slots}

The proposed on-chip metamaterial slotted antenna design is based on SIW structure [9, 10]. The on-chip integrated antenna in Fig.5 comprises a SIW, a $4 \times 4$ longitudinal and transverse slot array, and an interconnection line for the microstrip line and SIW. The top and bottom side of the SIW were constructed using $0.1 \mu \mathrm{m}$ aluminium pad layer and $0.5 \mu \mathrm{m} \mathrm{GaAs}$ layer with $\varepsilon_{r}$ : 12.94 and $\tan \delta: 0.006$, respectively. The bottom of the SIW is used as a ground-plane of the microstrip line. Sides of the SIW structure were created inserting metallic via-holes in the GaAs layer to the aluminium pad layer, and the longitudinal and transverse slot array were fabricated in the pad layer based on metamaterial concept [11-14] to improve the radiation performance parameters. The gap between the SIW and slot array was made $3 \lambda / 4$ purely because of the limitation of our fabrication facility.

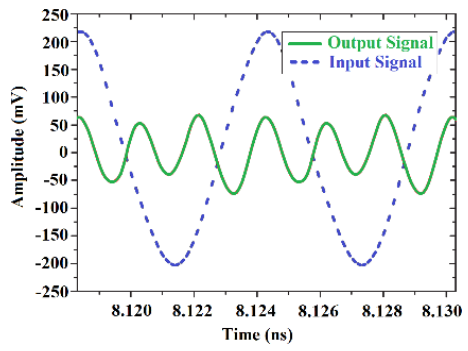

Fig.4. Simulated time-domain waveform of the frequency-tripler.

Dimensions of the SIW on-chip antenna, ground-plane, diameter of via holes, distance between via holes, length \& width of slots, distance between the slots in each row, length \& width of microstrip line, length \& width of interconnect line are $2 \times 1.5 \times 0.0001 \mathrm{~mm}^{3}, 2 \times 2 \times 0.0005$ $\mathrm{mm}^{3}, 0.025 \mathrm{~mm}, 0.077 \mathrm{~mm}, 3 \mathrm{~mm} \& 1 \mathrm{~mm}, 3 \mathrm{~mm}, 0.3 \mathrm{~mm}$ $\& 0.2 \mathrm{~mm}, 0.2 \mathrm{~mm} \& 0.070 \mathrm{~mm}$, respectively.

A SIW is used here as a high-pass structure [9] to filter out the unwanted $f_{0}$ and $2 f_{0}$ signals. Furthermore, the SIW is in close proximity to the waveguide structure that profoundly reduces unwanted surface waves and EM interference to neighbouring devices. The impedance of the SIW on-chip antenna can be adjusted by simply changing the width of the integrated waveguide structure and the microstrip line. Hence, the proposed antenna is easily conjugate matched with a tripler to minimize the loss. In addition, to increase the radiation properties of the SIW onchip antenna, a $4 \times 4$ array of longitudinal and transverse slots was added on top of the SIW. This approach extends the effective aperture area of the on-chip antenna to optimise radiation performances.

Fig. 6 shows the on-chip antenna operates over a frequency range from $300 \mathrm{GHz}$ to $310 \mathrm{GHz}$. The radiation performance parameters such as gain and efficiency plots are shown in Figs. 7 and 8. The minimum and maximum radiation gain and efficiency are $0.25 \mathrm{dBi}$ at $300 \mathrm{GHz} \&$ $1.75 \mathrm{dBi}$ at $305 \mathrm{GHz}$, and $46.12 \%$ at $310 \mathrm{GHz} \& 68.34 \%$ 
at $\sim 305 \mathrm{GHz}$, respectively. The average value of these parameters are $1.05 \mathrm{dBi}$ and $54.69 \%$, respectively.

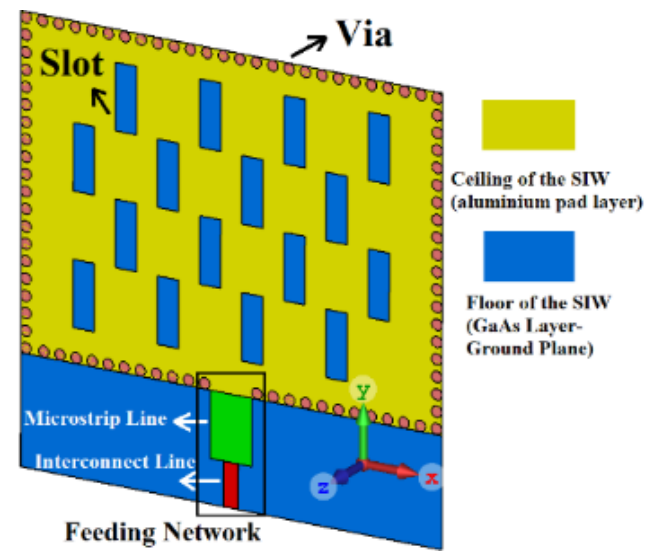

Fig.5. Configuration of the proposed on-chip antenna based on SIW and metamaterial technologies.

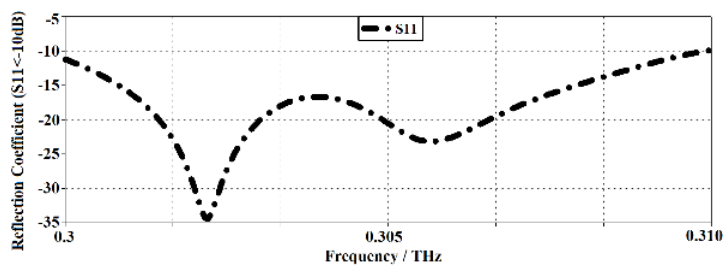

Fig.6. Reflection coefficient $\left(\mathrm{S}_{11}<-10 \mathrm{~dB}\right)$ of the proposed metamaterial on-chip antenna implemented on SIW.
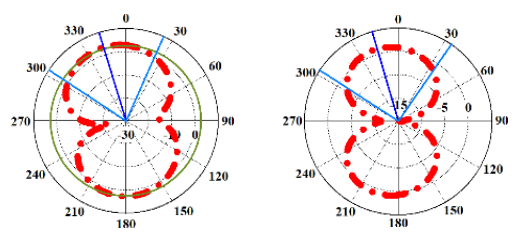

(a) $300 \mathrm{GHz}$
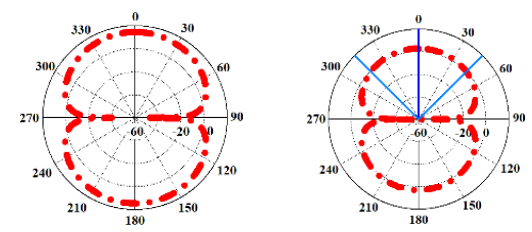

(a) $305 \mathrm{GHz}$
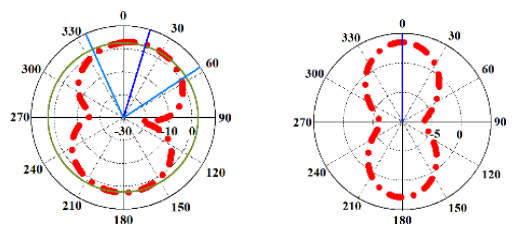

(a) $310 \mathrm{GHz}$

Fig.7. Radiation patterns of the proposed on-chip antenna at 300, 305, and $310 \mathrm{GHz}$. Left- and right-side show the E- and H-planes, respectively.
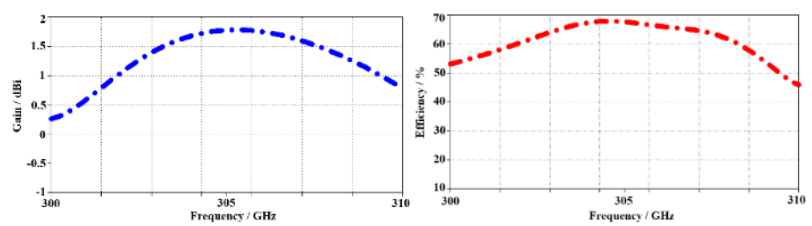

Fig.8. Radiation gain and efficiency curves of the on-chip antenna versus operational frequency.

\section{CONCLUSION}

An on-chip antenna which is fully integrated on GaAs based transceiver operates across 0.3-0.31 THz. The transceiver is constructed from a voltage-controller oscillator, a buffer amplifier, a modulator stage, a poweramplifier, a frequency-tripler, and an on-chip antenna. The on-chip antenna design was constructed using SIW technology and comprises $4 \times 4$ longitudinal and transverse array slots that are realized by metamaterial technology. The on-chip antenna has a minimum gain and efficiency of $0.25 \mathrm{dBi}$ and $46.12 \%$, respectively, over $300 \mathrm{GHz}$ to 310 $\mathrm{GHz}$, which enable it to be applicable for near-field active imaging applications at terahertz.

\section{ACKNOWLEDGMENTS}

This work is partially supported by innovation programme under grant agreement H2020-MSCA-ITN-2016 SECRET-722424 and the financial support from the UK EPSRC under grant EP/E022936/1.

\section{REFERENCES}

[1] P. D. Maagt, P. H. Bolivar, and C. Mann, "Terahertz Science, Enginneering and Systems - from Space to Earth Applications," in Encyclopedia of RF and Microwave Engineering, K. Chang, Ed. Wiley, 2005, pp. 5175-5194.

[2] M. Tonouchi, "Cutting-edge terahertz technology," Nature Photonics, vol. 1, pp. 97-105, Feb. 2007.

[3] A. Maestrini, J. S. Ward, J. J. Gill, C. Lee, B. Thomas, R. H. Lin, G. Chattopadhyay, and I. Mehdiand, "A frequency-multiplied source with more than $1 \mathrm{~mW}$ of power across the $840-900-\mathrm{GHz}$ band," IEEE Trans. Microw. Theory Tech., vol. 58, no. 7, pp. 3477-3490, Jul. 2010.

[4] K.B. Cooper, R.J.Dengler, N.Llombart, T. Bryllert, G. Chattopadhyay, E. Schlecht, J. Gill, C. Lee, A. Skalare, I. Mehdi, and P.H.Siegel, "Penetrating 3-D imaging at 4- and 25-m range using a submillimetrewave radar," IEEE Trans. Microw. Theory Tech., vol. 56, no. 12, pp. 2771-2778, Dec. 2008.

[5] E. Laskin, M. Khanpour, S. T. Nicolson, A. Tomkins, P. Garcia, A. Cathelin, D. Belot, and S. P. Voinigescu, "Nanoscale CMOS transceiver design in the 90-170-GHz range," IEEE Trans. Microw. Theory Tech., vol. 57 , no. 12 , pp. 3477-3490. Dec. 2009.

[6] B. Razavi, "A 300-GHz fundamental receiver for automotive radar," IEEE J. Solid-State Circuits, vol. 46, no. 4, pp. 894-903, Apr. 2011.

[7] E. Seok et al., "Progress and challenges towards terahertz CMOS integrated circuits," IEEE J. Solid-State Circuits, vol. 45, no. 8, pp. 15541564, Aug. 2010.

[8] E. Öjefors, J. Grzyb, Y. Zhao, B. Heinemann, B. Tillack, and U. R. Pfeiffer, "A $820 \mathrm{GHz}$ SiGe chipset for terahertz active imaging applications," ISSCC Dig. Tech. Papers, pp. 224-225, Feb. 2011.

[9] D. Deslandes and K. Wu, "Accurate modeling, wave mechanisms, and design considerations of a substrate integrated waveguide," IEEE Trans. Microw. Theory Tech., vol. 54, no. 6, pp. 2516-2525, 2006.

[10] L. Yan, W. Hong, G. Hua, J. Chen, K. Wu, and T. J. Cui, "Simulation and experiment on SIW slot array antennas," IEEE Microw. Wireless Compon. Lett., vol. 14, no. 9, pp. 446-448, 2004.

[11] M. Alibakhshi-Kenari, M. Naser-Moghadasi, R. A. Sadeghzadeh, B. S. Virdee and E. Limiti, "Periodic Array of Complementary Artificial Magnetic Conductor Metamaterials-Based Multiband Antennas for Broadband Wireless Transceivers," IET Microwaves, Antennas \& Propagation, vol.10, issue 15, 10 December 2016, pp.1682 - 1691.

[12] M. Alibakhshi-Kenari, M. Naser-Moghadasi, R. A. Sadeghzadeh, B. S. Virdee and E. Limiti, "Bandwidth Extension of Planar Antennas Using Embedded Slits for Reliable Multiband RF Communications", AEUE Elsevier-International Journal of Electronics and Communications, vol.70, issue 7, July 2016, pp. 910-919.

[13] R. A. Sadeghzadeh, M. Alibakhshi-Kenari and M. Naser-Moghadasi, "UWB Antenna Based on SCRLH-TLs for Portable Wireless Devices", Microwave and Optical Technology Letters, vol.58, issue 1, January 2016, pp.69-71.

[14] M. Alibakhshi-Kenari, A. Andújar and J. Anguera, "New Compact Printed Leaky-Wave Antenna with Beam Steering," Microwave and Optical Technology Letters, vol.58, issue 1, January 2016, pp. 215-217. 